\title{
A Cellular Automata Traffic Flow Model considering Bus Lane Changing Behavior with Scheduling Parameters
}

\author{
Zun-dong Zhang, ${ }^{1}$ Yan-fang Yang, ${ }^{2}$ Wenjiao Qi, \\ Abderrahim Chariete, ${ }^{3}$ and Xing-xiang Lin $^{4}$ \\ ${ }^{1}$ Beijing Key Lab of Urban Intelligent Traffic Control Technology, North China University of Technology, Beijing 100041, China \\ ${ }^{2}$ State Key Laboratory of Rail Traffic Control and Safety, Beijing Jiaotong University, Beijing 100044, China \\ ${ }^{3}$ OPERA Laboratory, University of Technology of Belfort-Montbéliard, 90000 Belfort, France \\ ${ }^{4}$ Xiamen Traffic Management Bureau, Xiamen 361003, China
}

Correspondence should be addressed to Zun-dong Zhang; zdzhang@ncut.edu.cn

Received 19 November 2014; Revised 22 January 2015; Accepted 23 January 2015

Academic Editor: Shuenn-Ren Cheng

Copyright (c) 2015 Zun-dong Zhang et al. This is an open access article distributed under the Creative Commons Attribution License, which permits unrestricted use, distribution, and reproduction in any medium, provided the original work is properly cited.

According to different driving behavioral characteristics of bus drivers, a cellular automata traffic model considering the bus lane changing behavior with scheduling parameters is proposed in this paper. Traffic bottleneck problems caused by bus stops are simulated in multiple lanes roads with no-bay bus stations. With the mixed traffic flow composed of different bus arrival rate, flow-density graph, density distribution graph, and temporal-spatial graph are presented. Furthermore, the mixed traffic flow characteristics are analyzed. Numerical experiment results show that the proposed model can generate a variety of complicated realistic phenomena in the traffic system with bus stops and provide theoretical basis for better using of traffic flow model.

\section{Introduction}

Because cellular automata have characteristics that its model is simple and can effectively reproduce the characteristics of the complex transport phenomena when computerimplemented; after it was introduced into the transport field, it has been rapidly developed [1-5]. As one of the major transportation systems, the operating status of public transportation system can affect the entire transportation system status, so the study of impact of public transport on road traffic flow has become a hot issue in recent years. Jia et al. [6] and Li et al. [7] compared dynamic characteristics of road traffic flow in harbor style and nonharbor style two different settings of bus station and analyzed the impact of bus proportion and the length of the bus station. Song and Zhao [8] and so forth used two-lane cellular automaton model simulated traffic bottleneck problems caused by interactions of entrance ramp and bus stops and discussed bus stops upstream and downstream two cases at the entrance ramp, respectively. Zhu [9] compared and analyzed urban traffic flow characteristics in the two-lane traffic system under three conditions: having bus lanes, intermittent bus specific road, and no bus lanes. Luo et al. [10] simulated and analyzed traffic flow of bus lines based on different departure rate and arrival rate.

However, the current bus behavior modeling lacks of further refinement on into and out station behaviors, that buses using a fixed stop time, first in first out (FIFO) principle, and so forth. But in reality, there are often cases that multiple buses simultaneously inbound, the actual stop time of bus is infected by passengers getting on and off, and buses last in first out (LIFO), and so forth. So the current bus behavior modeling is inconsistent with the actual situation.

In response to the problems existing in the model above, this paper takes multilane cellular automaton model as background, by defining bus parking traffic behavior rules in bus lanes and nonbay bus stations; the paper examines its impact on road traffic flows. 


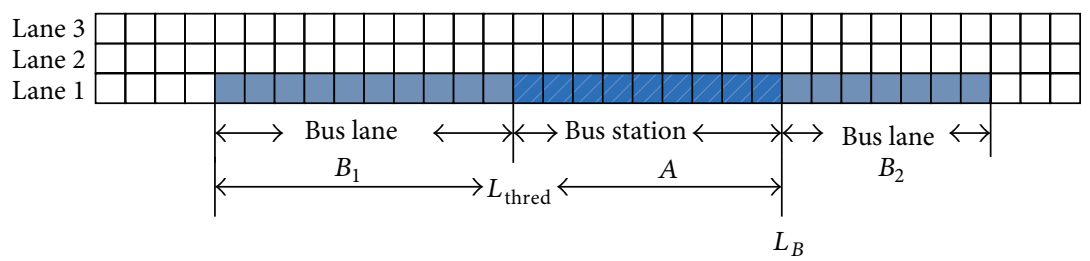

FIgURE 1: Schematic illustration of bus station.

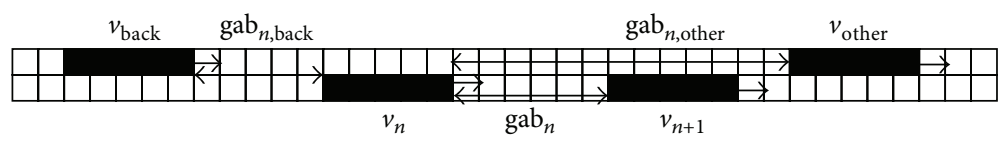

FIGURE 2: Schematic illustration of lane changing rules.

\section{The CA Model}

This paper discusses the nonharbor bus stops impact on traffic flow characteristics. By applying cellular automata model, sections that have bus stations are described as the system shown in Figure 1. The sections have three lanes (lane 1, lane 2, and lane 3); section length has $L$ cells. On the road, the vehicles are free to change. At the bus lanes, social vehicles are forced to change lanes into nonbus lanes to travel; buses on nondedicated lane need to change lanes into lane 1 to achieve getting into station and parking. The front part of bus station is set in $L_{B}$. The system considers social vehicles and buses two types; among them, social vehicles are divided into cars and coaches, and a car occupies 5 cells; a bus and a coach both occupy 10 cells. The maximum speed of cars is $v_{\max 1}=25$ cells; the maximum speed of buses and coaches is $v_{\max 2}=15$ cells.

2.1. Lane Change Rules. In addition to areas $A$ and $B_{1,2}$, vehicles can change lanes freely on the other sections; vehicle liberal lane changing rules are as follows:

$$
\begin{gathered}
\operatorname{gap}_{n}<\min \left\{v_{n}+1, v_{\text {max }}\right\}, \\
\operatorname{gap}_{n, \text { other }}>\operatorname{gap}_{n}, \\
\operatorname{gap}_{n, \text { back }}>v_{\text {max }} .
\end{gathered}
$$

Among them, gap ${ }_{n}$ gap $_{n, \text { other }}$, and gap ${ }_{n \text {,back }}$, respectively, represent empty cellular number between vehicle $n$ and original lane vehicle $n+1$, the front and behind vehicle in target lane (as shown in Figure 2). Particularly, when there are overlapping sections between vehicle $n$ and the front and behind vehicle in target lane, gap ${ }_{n, \text { other }}$ and gap gack $_{n \text {,ban }}$ can take negative number. gap $\sin _{n}<\min \left\{v_{n+1}, v_{\max }\right\}$, gap gather $_{n}>$ gap $_{n}$ indicates lane changing motivations and shows that the vehicle cannot continue acceleration driving on the current lane, whereas the target lane driving conditions are better than the current lane. gap ${ }_{n \text {,back }}>v_{\max }$ is safety conditions that guarantee the car will not collide with the behind car on the target lane after it changes the lane.

In areas $A$ and $B_{1,2}$, buses have special lane changing rules; specific rules are as follows.
2.1.1. In-Station Rules. Buses in the system have three states; use $k$ to denote, namely, (1) buses not parking in the stop, $k=0$; (2) buses parking in the stop, $k=1$; (3) buses have left the stop, $k=2$.

In the areas $A$ and $B_{1,2}$, the state of the bus is $k=0$; at this time the bus lane changing motivation is different to liberal lane changing motivation, whether driving conditions on lane 1 is good or bad; buses that have not stopped on lane 2 must change lanes to lane 1 (bus lanes) to realize getting into station and parking. At this time the mandatory lane changing rules are

$$
\begin{gathered}
\operatorname{gap}_{n, \text { other }} \geq v_{n}-v_{\text {other }}, \\
\operatorname{gap}_{n \text {,back }} \geq v_{\text {back }}-v_{n} .
\end{gathered}
$$

Equation (2) shows that as long as the current buses do not collide with the front and behind vehicle in target lane, lane changes can be achieved. Meanwhile, if buses on the right lane did not park, then they are prohibited from changing to left lanes before getting into station and parking.

If the Formula (2) is not meet, then the bus $n$ will send a signal to the behind vehicle on the target lane, behind vehicle on the target lane avoids the bus with a certain probability, then it meets the condition that

$$
\begin{gathered}
\operatorname{gap}_{n, \text { back }} \geq 0, \\
\operatorname{gap}_{n, \text { back }}<v_{\text {back }}-v, \\
\operatorname{rand}()<p, \quad p=1-\frac{L_{B}-x_{n}}{L_{\text {thred }}}, \\
v_{\text {back }}=\max \left\{0, \text { gap }_{n, \text { back }}\right\} .
\end{gathered}
$$

Among them, rand() represents the random number in $[0,1], x_{n}$ is the location of the bus $n, L_{\text {thred }}$ is the total length of the regional $A$ and $B_{1}$, and $p$ is the probability of behind car avoidance; as the bus $n$ is closer to $L_{B}$, the greater the probability of $p$, the higher the probability of collision avoidance.

If the bus $n$ on the left lane does not change lanes successfully until driving to $L_{B}$, then the bus will stop at this location, in order to wait for the opportunity to change lanes. 
Buses begin to stop next to the buses in front after entering the bus station, in the bus station; when the bus speed is 0 , we consider the bus stops and parking; passengers begin to get on and off, at this time, $k=1$.

2.1.2. Out-Station Rules. After the bus parking, $k=1$, stop time of bus $n$ is $T_{s}^{n}$; due to the different load factor of each bus, passengers getting on and off time is also different; assume the car parking time $T_{s}^{n}$ is uniformly distributed. When the parking time meets $t_{\text {stop }}^{n}=T_{s}^{n}$, bus $n$ completes parking, at this time the status of bus $n$ changes to $k=2$; bus $n$ can continue moving forward.

If the vehicle $n+1$ in front of bus $n$ is still parking, then bus $n$ looks for changing lane chances in probability $p_{\text {change; }}$; otherwise it will still remain in the place; parking cumulative time is $t_{\text {stop }}^{n}=T_{s}^{n}+1$; then the changing lane conditions are

$$
\begin{gathered}
\operatorname{gap}_{n, \text { back }} \geq 0 \text {, gap }_{n, \text { back }}>v_{\text {back }}, \\
\operatorname{rand}()<p_{\text {change }}, \quad p_{\text {change }}=1-\frac{T_{\max }-t_{\text {stop }}^{n}}{T_{\max }} .
\end{gathered}
$$

Among them, $\operatorname{rand}()$ represents the random number in $[0,1]$ and $T_{\max }$ is the maximum time bus drivers can wait for; the longer the wait time, the greater the probability of the bus changing to the left lanes. After pulling out of the bus station, buses have finished parking; in area $B_{2}$ they change lanes to liberal lane, at this time, and social vehicles cannot change lanes to bus lanes.

2.2. The Forward Movement Model. Forward movement rules of vehicles are improved on the basis of NaSch model [11]; evolution rules are as follows:

(1) acceleration: $v_{n} \rightarrow \min \left(v_{n}+1, v_{\max }\right)$;

(2) deceleration: $v_{n} \rightarrow \min \left(v_{n}, d_{n}\right)$;

(3) for nonstop buses, decelerations for the purpose of changing lanes, pit stop, and parking $(k=0): v_{n} \rightarrow$ $\min \left\{v_{n}, d_{B n}\right\}$;

(4) random slow (with probability $q): v_{n} \rightarrow \max \left(v_{n}-\right.$ $1,0)$;

(5) location update: $x_{n} \rightarrow x_{n}+v_{n}$.

Among them, $v_{n}$ represents the number of empty cells between the $n$ vehicle and the $n+1$ vehicle, $d_{n}=x_{n+1}-x_{n}-l_{n+1}$ denotes the number of empty cells between the $n$ vehicle and the front vehicle (namely, the $n+1$ vehicle), and $l_{n+1}$ is the length of the $n+1$ vehicle, that is, the number of cells it occupies.

2.3. Boundary Conditions. The system uses the open boundary conditions, assuming that the left-most cell of each lane corresponds to $x=1$, rightmost cell corresponds to $x=$ $L, x_{\text {last }}$ denotes head position of leftmost vehicle, and $x_{\text {lead }}$ denotes head position of rightmost vehicle; in time step $t \rightarrow t+1$, when the update of lane location on the road is completed, update the value of $x_{\text {last }}$ and $x_{\text {lead }}$. If $x_{\text {last }}-l-1>0$, then the vehicles in speed $\min \left\{x_{\text {last }}-l-1, v_{\max }\right\}$ enter cells in probability $\alpha$; inflow vehicles set buses according to a certain proportion $R$.

In the exit of lane, the system uses fully open boundary conditions; that is, when the head position of vehicle meets $x_{\text {lead }}>L$, the leading vehicle will exit the system, and the next vehicle will become the leading vehicle and will travel without hinder.

\section{Simulation and Analysis}

The system uses three lane cellular automaton models; buses, coaches, and cars are randomly placed at the initial state. Assuming road length is $L=2000$ cells, departure probability of the system is $\alpha=0.9$, system random slow probability is $q=0.1$, head position of bus is $L_{B}=1000$, the length of bus is 50 cells, namely, it can accommodate five buses at the same time, special lane changing interval length is $L_{\text {thred }}=120$ cells, and bus stop time is uniformly distributed to $[30,60]$. Simulation length is 20000 times; select the simulation data in the later 15000 times and make statistics of them.

Select the established bus into and out station model in [8]; for comparison, compare their simulation results, in which the proportion of buses is $R=0.2$. First, make statistics of the traffic density of the system; its statistical formulas are as follows:

$$
\begin{gathered}
\rho=\frac{5\left(N_{1}+2 N_{2}\right)}{4 L}, \\
v=\frac{N_{1} \bar{v}_{1}+2 N_{2} \bar{v}_{2}}{N_{1}+2 N_{2}}, \\
q=\rho v=\frac{5\left(N_{1}+2 N_{2}\right)}{4 L} \cdot \frac{N_{1} \bar{v}_{1}+2 N_{2} \bar{v}_{2}}{N_{1}+2 N_{2}} .
\end{gathered}
$$

Among them, $N_{1}$ is the number of small cars in the systems; $\mathrm{N}_{2}$ is the number of big cars and buses in the systems, $\bar{v}_{1}$ is the average speed of small cars in the systems, and $\bar{v}_{2}$ is the average speed of big cars and buses in the systems.

Flow and density comparative results of the two models are shown in Figure 3. As can be seen from Figure 3, at the same density value, the flow in the improved simulation model is bigger than that in the model of [8]. It mainly regulates the avoidance behavior of into and out station, plus the behavior of outbound advance; it also reduces the waiting time of buses getting into (or out) station; thereby it reduces the congestion probability of bus stop in the upstream.

Figure 4 is density-distribution diagram. As can be seen from the chart, the peak of solid line appears in the location of the bus station (950-1000 cells); it reflects the principles that haven't get into station buses have priority to get into station. When there is vacancy in bus station, buses which are not into station on adjacent lanes can choose to get into station and stop; however, the peak of dotted line appears in the pit mouth of bus station (950-900 cells), that buses must wait in line at the bus stop line. The downstream density of solid line is higher than the density of the dotted line; due to buses getting into station and parking buses timely, it increases downstream flow of buses. From the figure, 


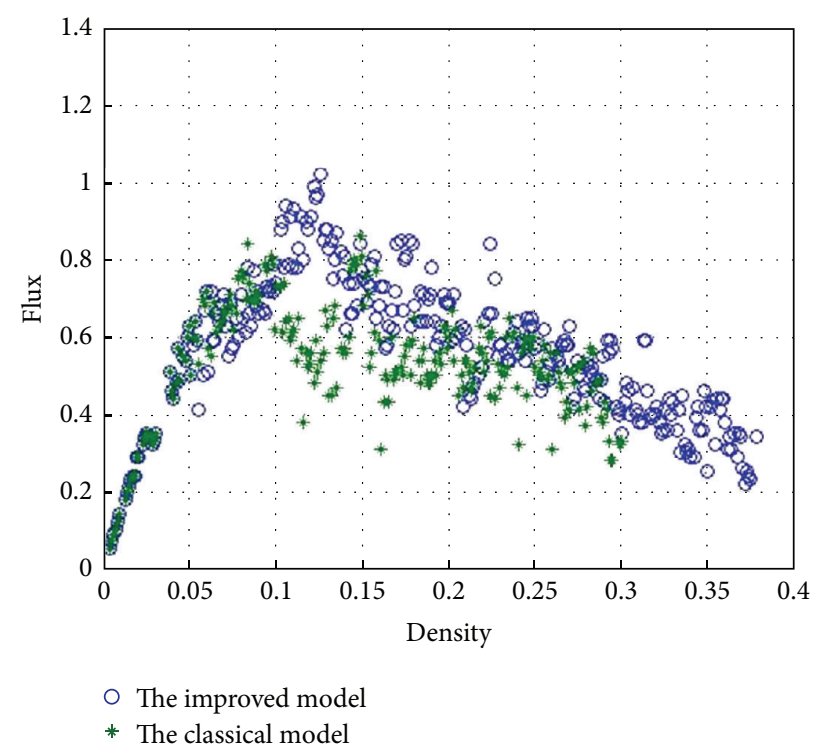

Figure 3: Flux-density diagram. "o" is the value of proposed model; “*” is the value of the classical model cited in [8].

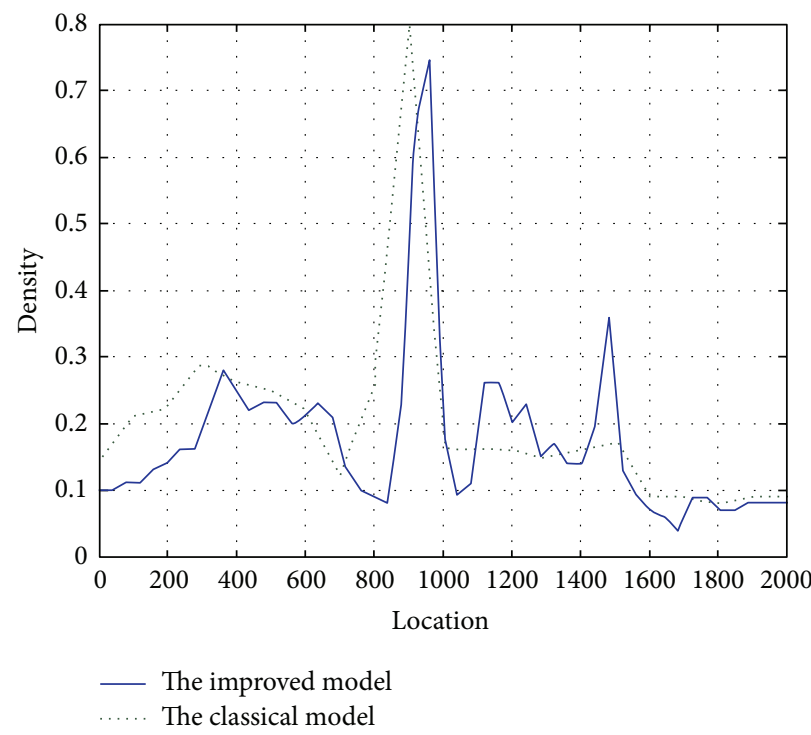

(a)

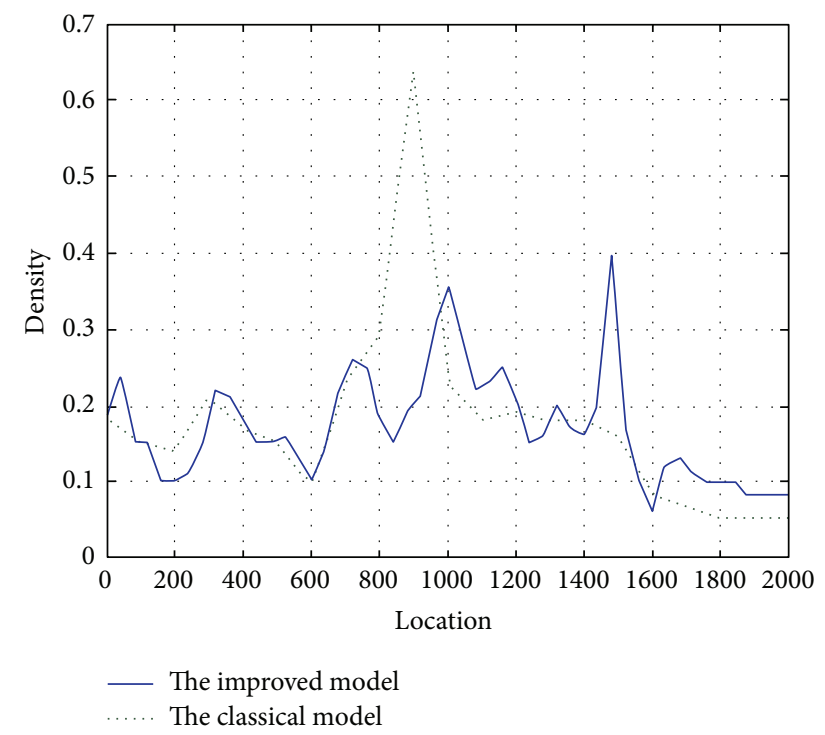

(b)

FIGURE 4: Density-distribution diagrams. (a) shows the density distribution in lane 1 and (b) shows the density distribution in lane 2.

the density distribution of solid line is much more evenly than the density distribution of dotted line. Density peak of dotted line appears at the upstream of bus station; this is mainly because unsuccessful changing lane buses are queuing stint here.

In summary, the bus station processing ability in improved model should be higher than that in the original model.

Select bus proportion $R=0.15,0.2$, and 0.3 , respectively, and analyze its traffic flow characteristics. Firstly, analyze the influences of bus proportion on bus getting into station behavior, as shown in Figures 5 and 6.
The black lines in the first column indicate vehicles at the location of bus station, which successfully change lanes from other lanes and get into station meanwhile to vehicles at the bus lane; the black line in the second column indicates vehicles that change into bus lanes from lane 2 at the location of bus station, while the black dots indicate second successfully changing lane vehicles, namely, from lane 3 swap to lane 2, and buses that finally swap to the bus lane (lane 1).

As can be seen from the comparison of the experimental results, when the bus proportion is low, cases that buses get into station simultaneously are very few; namely, buses complete lane change in front of bus stations; vehicles that 


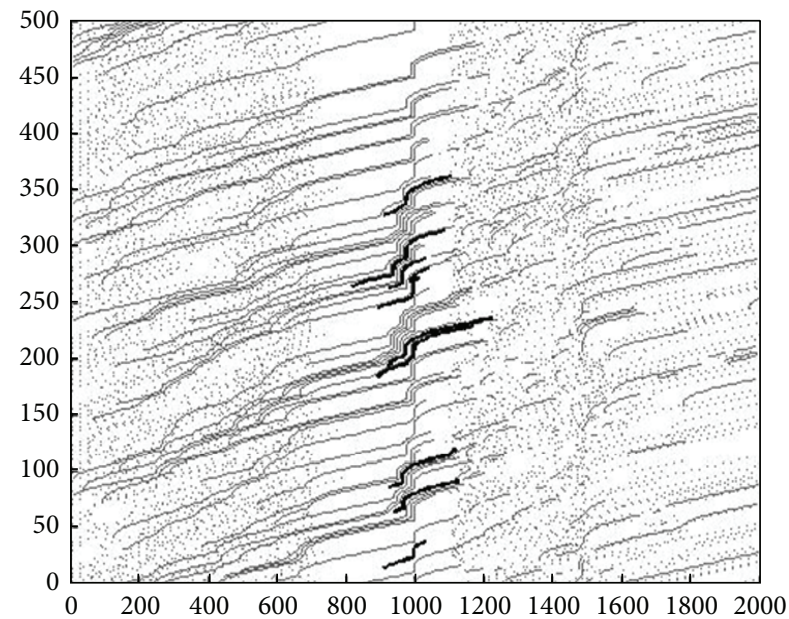

(1)

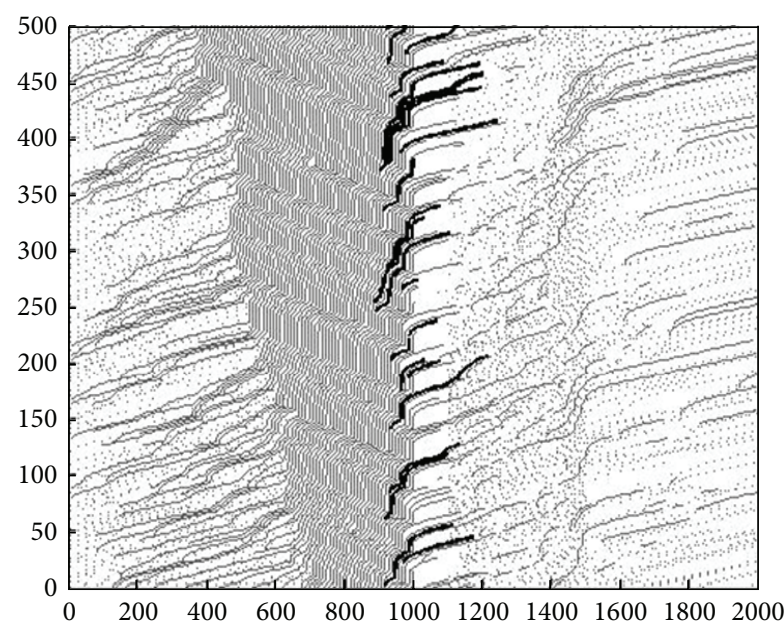

(2)

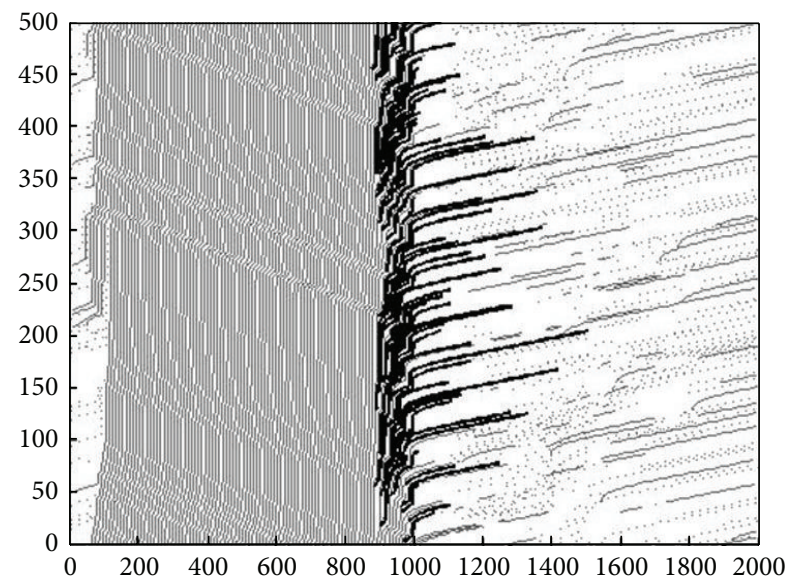

(3)

(a)

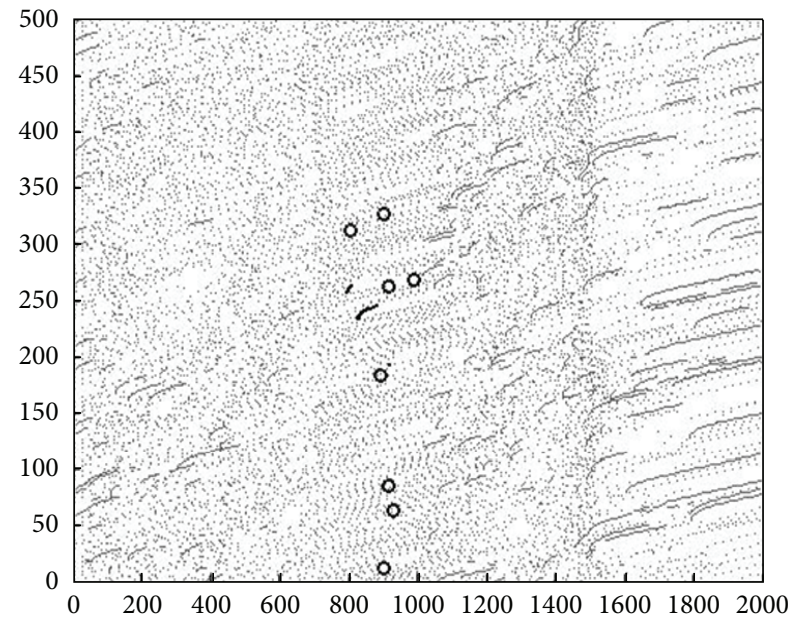

(1)

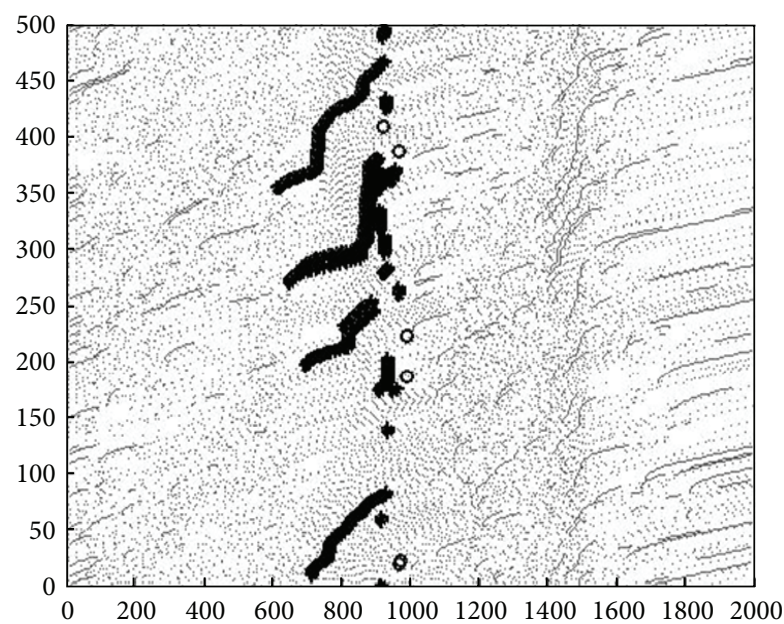

(2)

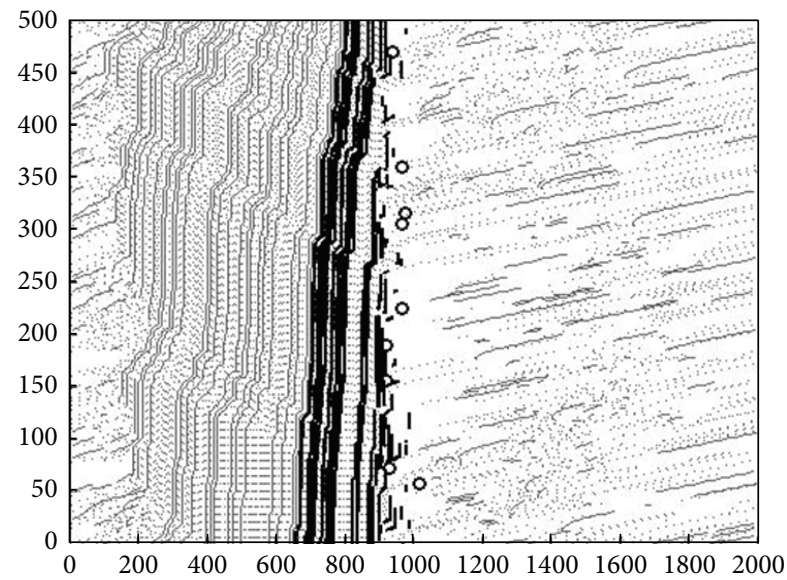

(3)

(b)

FIGURE 5: Space-time diagram over 500 time steps. Horizontal axis is for position and vertical axis is for time. (a) is for lane 1 and (b) are for lane 2. The first row: $R=0.15$, the second row: $R=0.2$; the third row: $R=0.3$. 


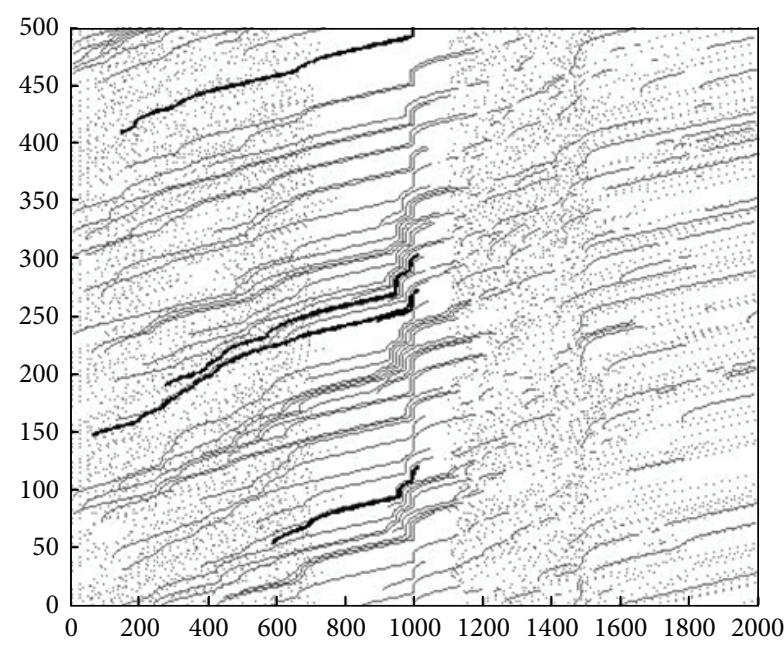

(1)

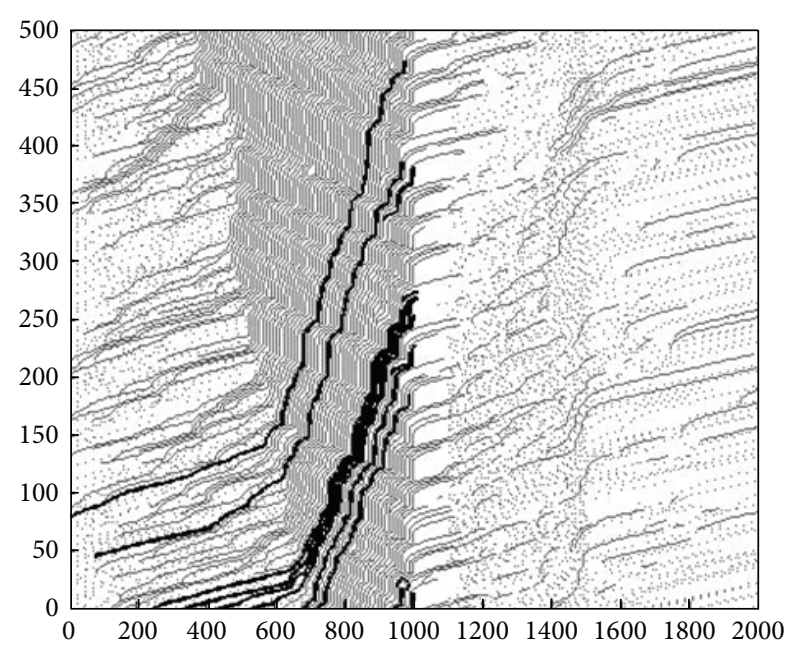

(2)

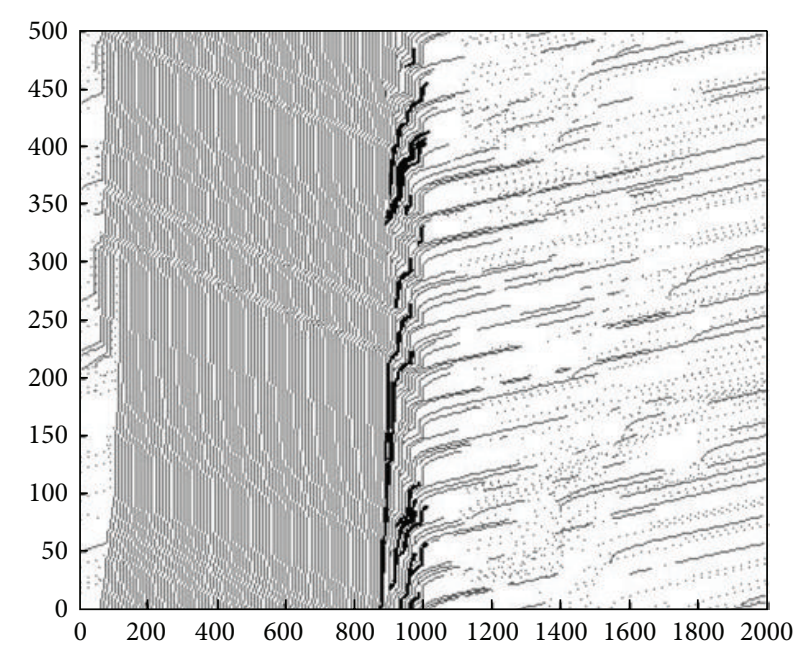

(3)

Figure 6: Space-time diagram for pulling out of bus stops. (1) $R=0.15$, (2) $R=0.2$, and (3) $R=0.3$.

simultaneous to buses get into station are mainly vehicles that outside bus lanes (lane 1). When lane change to the bus lane is not successful, buses can only queue and wait to get into station on lane 2 . With the increase of the bus proportion, unsuccessful probability of buses changing lanes into bus lane becomes higher; buses on lane 2 can only move forward while waiting on the original lane, so cases that get into station at the same time increase.

Secondly, analyze the influence of bus proportion on bus getting out station behavior, as shown in Figure 6 . With the increase of bus proportion, the proportion of buses getting into station increases, and the proportion of outbound advance also increases.

\section{Conclusions}

This paper simulates and studies the nonbay bus station system on sections under the open boundary conditions and defines the buses getting into/out station rules and deeply studies the impact of buses getting into/out station behavior on traffic flow. It analyzes traffic flow characteristics by using flow and density diagram, density distribution, and spacetime diagram.

As can be seen from the simulation results, the avoidance behavior rule, buses meanwhile stint and advance outbound rules; it reduces the chances of congestion in the system caused by buses queue and wait to get into station, and it is more suitable to the reality. Meanwhile, the probability of occurrence of the above acts increases as buses proportion increases.

\section{Conflict of Interests}

All the authors of the paper declare that there is no conflict of interests regarding the publication of this paper.

\section{Acknowledgments}

This paper is supported by the Scientists Training Project on Urban Road Traffic Intelligent Control (no. 
PXM2014_014212_000053) and the Key Project of the Ministry of Public Security of China (no. 201302ZDYJ012) and the National Science \& Technology Pillar Program of China (no. 2014BAG01B02).

\section{References}

[1] B. S. Kerner and S. L. Klenov, "Spatial-temporal patterns in heterogeneous traffic flow with a variety of driver behavioural characteristics and vehicle parameters," Journal of Physics A: Mathematical and General, vol. 37, no. 37, pp. 8753-8788, 2004.

[2] J.-X. Ding, H.-J. Huang, and T.-Q. Tang, "Random noise in driving behavior based on cellular automata," Journal of Jilin University (Engineering and Technology Edition), vol. 39, supplement 2, pp. 66-70, 2009.

[3] L.-J. Peng and R. Kang, "One-dimensional cellular automaton model of traffic flow considering drivers' features," Acta Physica Sinica, vol. 58, no. 2, pp. 830-835, 2009.

[4] S. Kukida, J. Tanimoto, and A. Hagishima, "Analysis of the influence of lane changing on traffic-flow dynamics based on the cellular automaton model," International Journal of Modern Physics C, vol. 22, no. 3, pp. 271-281, 2011.

[5] X.-M. Liu and L. Wang, "Cellular automaton traffic flow model considering on-street parking," Journal of Jilin University, vol. 42, no. 2, pp. 327-333, 2012.

[6] B. Jia, X.-G. Li, R. Jiang, and Z.-Y. Gao, "The influence of bus stop on the dynamics of traffic flow," Acta Physica Sinica, vol. 58, no. 10, pp. 6845-6851, 2009.

[7] Q.-D. Li, L.-Y. Dong, and S.-Q. Dai, "Investigation on traffic bottleneck induce by bus stopping with a two-lane cellular automaton model," Acta Physica Sinica, vol. 58, no. 11, pp. 75847590, 2009.

[8] Y.-K. Song and X.-M. Zhao, "Combined bottleneck effect of on-ramp and bus stop in a cellular automaton model," Chinese Physics B, vol. 18, no. 12, pp. 5242-5248, 2009.

[9] H. B. Zhu, "Numerical study of urban traffic flow with dedicated bus lane and intermittent bus lane," Physica A: Statistical Mechanics and its Applications, vol. 389, no. 16, pp. 3134-3139, 2010.

[10] Y.-J. Luo, B. Jia, X.-G. Li, C. Wang, and Z.-Y. Gao, "A realistic cellular automata model of bus route system based on open boundary," Transportation Research Part C: Emerging Technologies, vol. 25, pp. 202-213, 2012.

[11] K. Nagel and M. Schreckenberg, "A cellular automata model for traffic flow," Journal de Physique, vol. 2, pp. 2221-2229, 1992. 


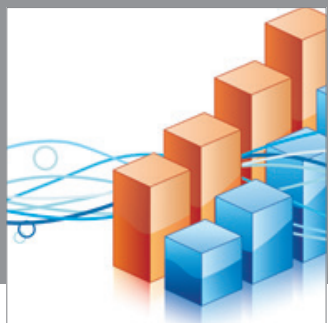

Advances in

Operations Research

mansans

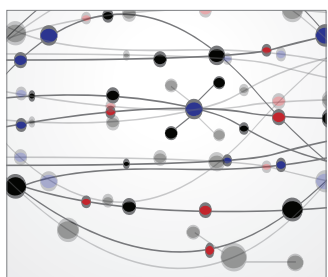

The Scientific World Journal
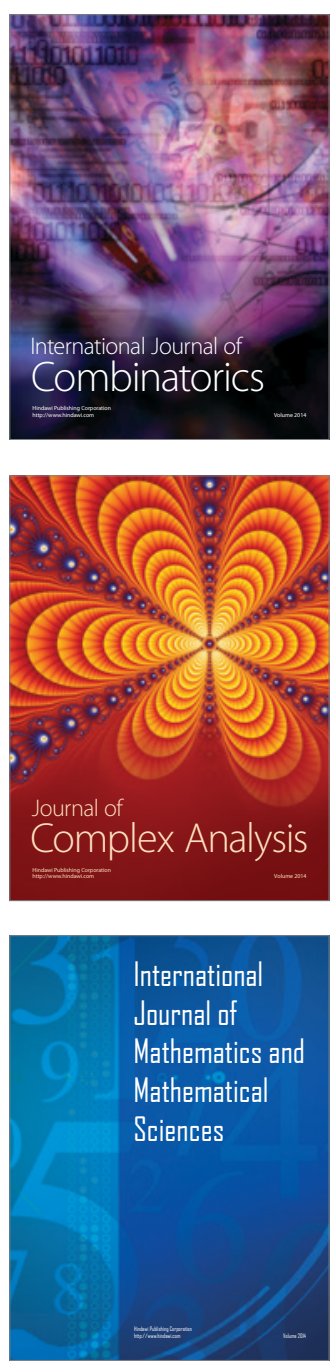
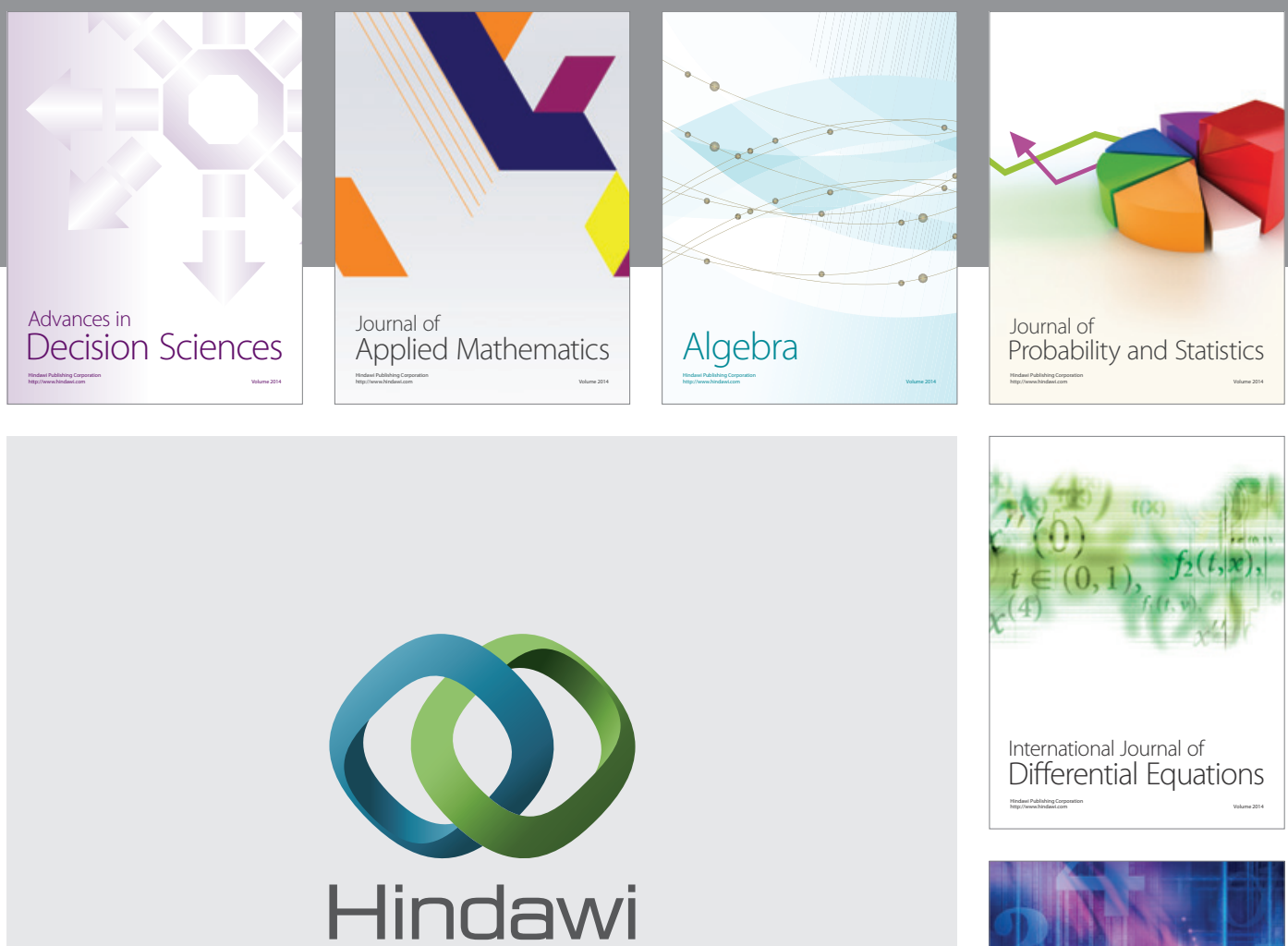

Submit your manuscripts at http://www.hindawi.com
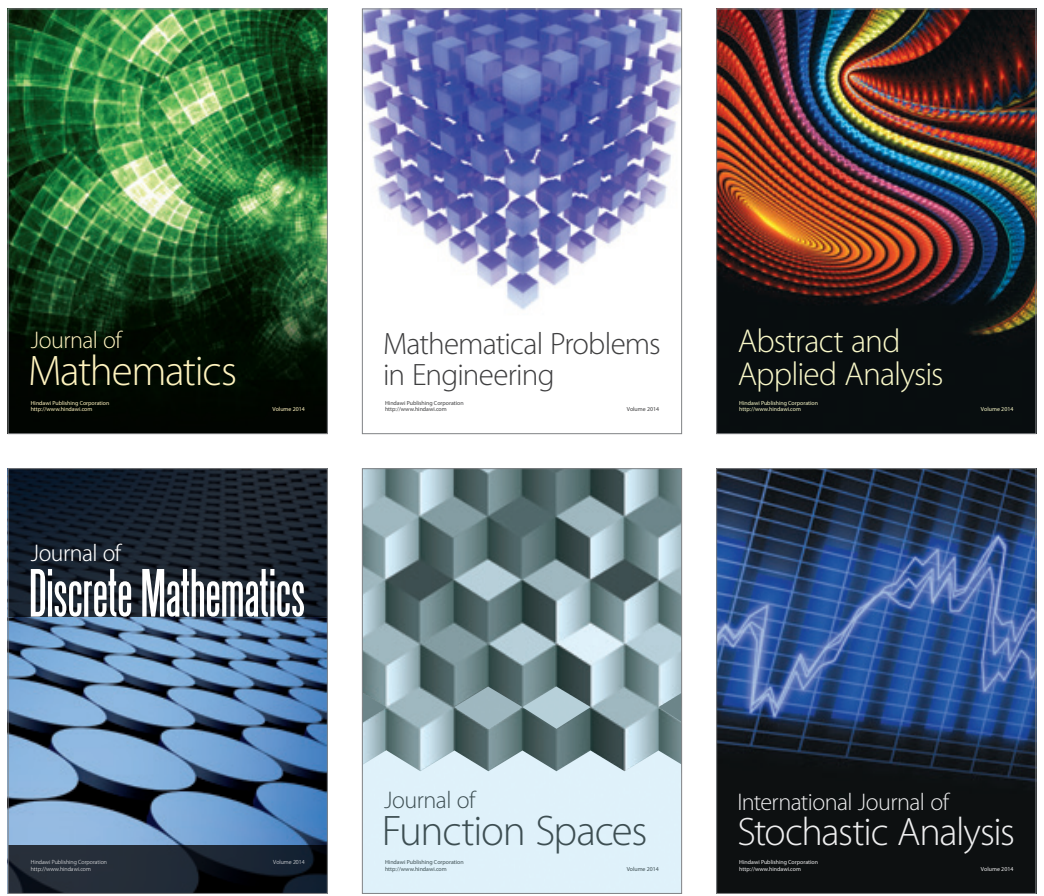

Journal of

Function Spaces

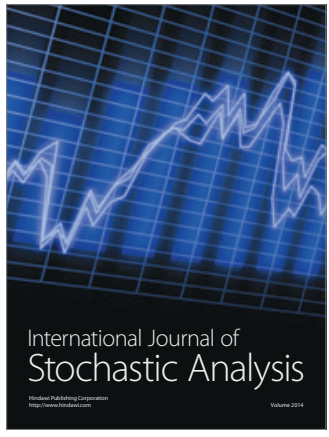

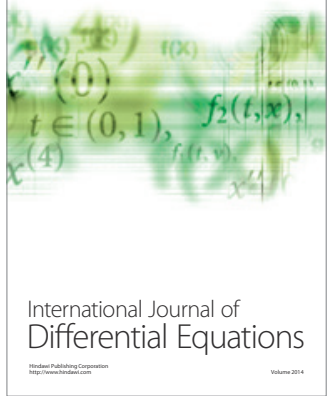
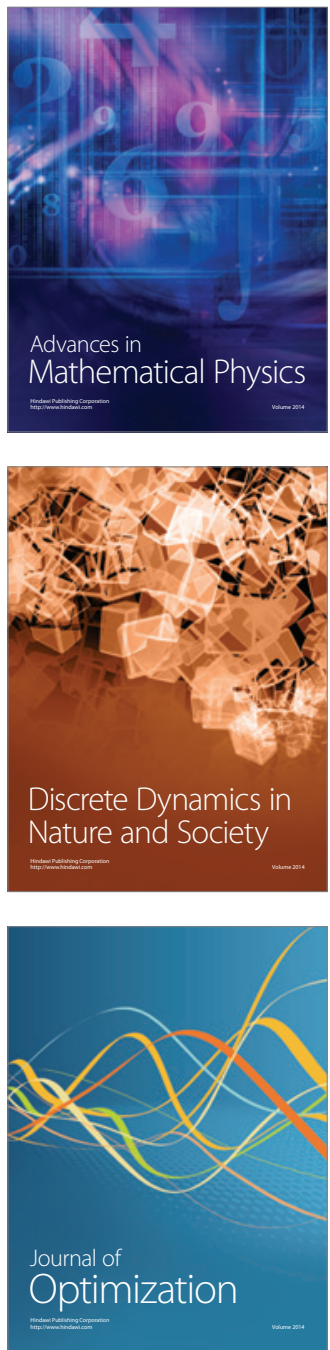